\title{
Specific miRNA expression profile in the blood serum of cardiac myxoma patients
}

\author{
LIANGLIANG YAN, JIAHUI LI, QINGSONG WU and LIANGWAN CHEN
}

\author{
Department of Cardiac Surgery, Union Hospital of Fujian Medical University, Fuzhou, Fujian 350001, P.R. China
}

Received October 27, 2017; Accepted May 22, 2018

DOI: $10.3892 /$ ol.2018.9209

\begin{abstract}
The profile of differentially expressed microRNAs (miRNAs) in the serum of patients with cardiac myxoma $(\mathrm{CM})(\mathrm{n}=30)$ and healthy people $(n=30)$ was studied using miRNA microarray analysis. The expression of the candidate miRNAs was validated by reverse transcription-quantitative polymerase chain reaction (RT-qPCR) in the serum of $30 \mathrm{CM}$ patients and 30 healthy control individuals. TargetScan, PicTar and miRanda were used to predict the possible target gene of miR-320a. The Gene Ontology database and Kyoto Encyclopedia of Genes and Genomes database were used to enrich the functions and signaling pathways of the target genes, respectively. The results showed that 4 differentially expressed miRNAs were identified, the expression levels of miR-320a and miR-1249-5p were upregulated, and those of miR-634 and miR-6870-3p were downregulated in $\mathrm{CM}$ patients $(\mathrm{P}<0.05)$. The expression levels of miR-320a and miR-634 selected for verification by RT-qPCR were in high concordance with the results of microarray analysis. Through bioinformatics, we identified 487 target genes predicted from miR-320a, that were mostly enriched in the bone morphogenetic protein signaling pathway, nicotinamide adenine dinucleotide pathway and de novo ceramide biosynthetic pathway. In our study, we reported for the first time the circulating miRNA profile of CM patients and suggested that miR-320a may participate in CM development through the ceramide signaling pathway.
\end{abstract}

\section{Introduction}

Cardiac myxoma (CM) is the most clinically common primary cardiac benign tumor, its incidence accounts for more than $50 \%$ of cardiac tumors (1). CM frequently occurs in the middle age and is more common in females than in males but rarely seen in children, in whom it accounts for $15 \%$ of cardiac

Correspondence to: Professor Liangwan Chen, Department of Cardial Surgery, Union Hospital of Fujian Medical University, 29 Xin Quan Road, Fuzhou, Fujian 350001, P.R. China

E-mail: chenliangwan1@sina.com

Key words: cardiac myxoma, microRNA, aligent miRNA expression profiles chip, qPCR, miR-320a tumors (2,3). CM occurs anywhere in the heart, but arises most commonly in the left atrium $(4,5)$ with occasionally on the right atrium, right ventricle and the left ventricle $(5,6)$. It can cause mild constitutional symptoms, such as fever, weight loss, myalgia, skin flushing or arthralgia, to serious hemodynamic derangement, depending on its location and size, which can lead to disastrous embolic symptoms. Therefore, early diagnosis and treatment are necessary, and once the diagnosis is established, surgery should be performed without delay. However, recurrence after surgical excision occurs in $2 \%$ of the cases (7). CM can be easily misdiagnosed and treatment can be delayed due to its complicated clinical manifestations with no specific clinical performance. Echocardiography is the most commonly used method for the detection of CM. The diagnosis rate has been improved obviously since it was widely used in the clinic. However, echocardiography has limitations due to low sensitivity and specificity, lengthy diagnostic time, and technical complexity. Therefore, diagnostically sensitive and specific markers for early uncomplicated CM detection are urgently needed.

A microRNA (miRNA/miR) is an endogenous small single-stranded non-coding RNA molecule with a length of $\sim 22$ nucleotides. It can pair with the 3'UTR of a target gene's mRNA, and inhibit the translation of the target genes or induce the degradation of mRNA (8). miRNAs are involved in numerous cancer-relevant processes, such as migration, proliferation and apoptosis $(9,10)$. Aberrant expression of miRNA has been reported in various malignancies such as gastric cancer (11) and breast cancer (12), and thus, their alteration can cause malignant transformation. Recent evidence demonstrated that circulating miRNAs can be regarded as reliable biomarkers for the detection of various cancers and other diseases due to their stability and ease of detection (13). However, miRNA in the serum of patients with CM remains unexplored, and the pathogenesis of CM is not very clear. Therefore, in the present study, we aimed to identify differentially expressed miRNAs in the serum of patients with CM and analyzed their potential role in CM development.

\section{Materials and methods}

Patients. Thirty patients with CM who were hospitalized in Fujian Medical University Union Hospital (Fuzhou, China) from October 2015 to May 2016 were recruited. Thirty healthy cases were also recruited. Of the 30 patients with CM, 10 were 
male and 20 were female, and their age ranged from 17 to 88 , with average age of 58.5. In the control group, 10 were male and 20 were female, and their age ranged from 18 to 74 with an average age of 55.6. No significant difference was found $(\mathrm{P}>0.05)$ in the general characteristics, such as gender and age, between the CM and healthy groups. This study was approved by the Ethics Committee of Fujian Medical University Union Hospital (Fuzhou, China) and informed consent was provided by each patient.

Serum preparation and total RNA isolation. Blood was collected from each subject into tubes without anticoagulant and placed at $4^{\circ} \mathrm{C}$ for $2 \mathrm{~h}$. The samples were then centrifugated at $4,000 \mathrm{x} \mathrm{g}$ for $15 \mathrm{~min}$, and the serum that was separated was stored at $-80^{\circ} \mathrm{C}$ until use. The 30 patients with $\mathrm{CM}$ were randomly assigned into three equal groups of 10 subjects each, then $100 \mu \mathrm{l}$ of the serum form each group were pooled to generate a serum pool (14) labeled as samples A, B and E. Similarly, the samples from the healthy group were also grouped, and the serum pools were labeled as samples a, b and e. We named the groups randomly, these names have no meaning.

Total RNA from the serum was extracted and purified using the miRNeasy Mini kit (Qiagen, Inc., Valencia, CA, USA). The concentration and quality of the RNA were measured using a NanoDrop 1,000 Spectrophotometer (Thermo Fisher Scientific, Inc., Waltham, MA, USA). RNA samples were subjected to electrophoresis with $1.4 \%$ agarose-formaldehyde gels stained with ethidium bromide to verify their integrity. The quality control criteria of RNA were $28 \mathrm{~S} / 18 \mathrm{~S}>1.5$ and A260/A280 $>1.8$.

MiRNA expression profiling. The RNA with high purity (A260/A280 >1.8) and high quality $(28 \mathrm{~S} / 18 \mathrm{~S}>1.5)$ was used for the microarray experiments. The miRCURY Hy3/Hy5 Power Labeling kit (Exiqon, Vedbaek, Denmark) was used according to the manufacturer's guideline for miRNA labelling. One microgram of each sample was 3-end-labeled with Hy3TM fluorescent label by using T4 RNA ligase. The mixture was incubated for $30 \mathrm{~min}$ at $37^{\circ} \mathrm{C}$, and the reaction was terminated by incubation for $5 \mathrm{~min}$ at $95^{\circ} \mathrm{C}$. Then, $3.0 \mu \mathrm{l}$ of labeling buffer, $1.5 \mu \mathrm{l}$ of fluorescent label (Hy3TM), $2.0 \mu \mathrm{l}$ of DMSO, and $2.0 \mu \mathrm{l}$ of labeling enzyme were added into the mixture. The labeling mixture was incubated for $1 \mathrm{~h}$ at $16^{\circ} \mathrm{C}$, and the reaction was terminated by incubation for $15 \mathrm{~min}$ at $65^{\circ} \mathrm{C}$. After stopping the labeling procedure, the Hy3-labeled samples were hybridized to the Agilent miRNA microarray chip. Following the hybridization, the slides were achieved, washed several times by using a Wash Buffer kit (Exiqon), and finally dried by centrifugation for $5 \mathrm{~min}$ at $400 \mathrm{x} \mathrm{g}$. Then the microarray was scanned using the GenePix 4000B microarray scanner (Axon Instruments, Foster City, CA, USA). Data were extracted using the Agilent Feature Extraction Software and analyzed using the GeneSprint 13.0 software (Agilent Technologies, Inc., Santa Clara, CA, USA). All raw data in control and CM group was processed with 'percentile' method for data normalization, and FC (fold-change) was calculated using these normalized datas. The miRNAs with $\mathrm{FC} \geq 2.0$ and $\mathrm{P}<0.05$ were considered significant, the choice of cut-off was based on previous studies (15-18). This section was accomplished by Beijing CapitalBio Corporation (Beijing,
China). The data in healthy group was used as control for the comparisons.

Validation of the differentially expressed miRNAs by quantitative reverse transcription-quantitative polymerase chain reaction ( $R T-q P C R)$. Two selected candidate miRNAs identified from the serum samples of the 30 patients with CM and 30 healthy controls were validated by RT-qPCR. $1 \mu \mathrm{l}$ of purified total RNA was reverse transcribed using a miscript II RT kit (Qiagen, Courtaboeuf, France) according to the manufacturer's instructions. The qPCR assay was performed using reagents from the SYBR Green Real-Time PCR kit (Takara Biotechnology Co., Ltd., Dalian, China), and the reaction mix was composed of $10 \mu \mathrm{l}$ SYBR Premix Ex Taq II, $0.8 \mu \mathrm{l}$ PCR forward primer (Table I), $0.8 \mu \mathrm{l}$ UnimiR qPCR primer, $2 \mu \mathrm{l}$ cDNA, $0.4 \mu \mathrm{l}$ ROX reference dye, and $6 \mu$ l deionized $\mathrm{H}_{2} \mathrm{O}$. The reactions were incubated in a 48 -well optical plate at $95^{\circ} \mathrm{C}$ for $30 \mathrm{sec}$, followed by 40 cycles of $95^{\circ} \mathrm{C}$ for $5 \mathrm{sec}$, and $60^{\circ} \mathrm{C}$ for $34 \mathrm{sec}$. The Cquantification cycle $(\mathrm{Cq})$ was determined by using the default threshold settings. All experiments were performed in triplicate and repeated three times. RT-qPCR was performed in Mx3000P (Stratagene, La Jolla, CA, USA). U6 small-nuclear RNA (U6 snRNA) was used as a reference gene to normalize the expression of the miRNA. The mature sequences for all miRNAs were acquired from miRBase (http://www.mirbase. org/). The primer sequences used for the miRNA analysis are designed based on these mature sequences and shown in Table I. Relative expression was analyzed using the method of $2^{-\Delta \Delta \mathrm{Cq}}$ (19).

Bioinformatics analysis. The target genes of miR-320a were predicted using three miRNA databases including miRanda (http://mirdb.org/miRDB/), TargetScan (http://www. targetscan.org/) and PicTar (http://pictar.mdc-berlin.de). The target genes that were uniformly predicted by the three databases were selected. The Gene Ontology (GO) database (http://www.geneontology.org/) was employed to enrich the functions of miR-320a target genes. Fisher's exact test was then employed to calculate the P-value for each function. A function with $\mathrm{P}$-value $<0.05$ was considered as significant. The Kyoto Encyclopedia of Genes and Genomes (KEGG) database (http://www.kegg.jp/) was used to explore the relevant signaling pathways of the target genes of miR-320a. Fisher's exact test was then employed to calculate the P-value for each pathway. A pathway with P-value $<0.05$ was considered as significant.

Statistical analysis. All data were shown as mean \pm standard deviation, and analyzed using the SPSS 20.0 software (SPSS, Inc., Chicago, IL, USA). The differential expression levels between two groups was analyzed using t-test for validation. $\mathrm{P}<0.05$ was considered to indicate a statistically significant difference. P-values were corrected for multiple testing by false discovery rate (FDR). Clustering analysis of miRNA based on KK-means algorithm.

\section{Results}

Differentially expressed miRNAs in the serum by miRNA microarray analysis. We performed a microarray analysis to 
Table I. miRNA-specific Primers Used in the RT-qPCR.

miRNA Primer sequences (5'-3')

$\begin{array}{ll}\text { miR-320a } & \text { Forward:5'-GCGAAAAGCTGGGTTGAGA-3' } \\ & \text { Reverse:5'-AGTGCAGGGTCCGAGGTATT-3' } \\ \text { miR-634 } & \text { Forward: 5'-CGAACCAGCACCCCAACT-3' } \\ & \text { Reverse:5'-AGTGCAGGGTCCGAGGTATT-3' } \\ \text { U6 } & \text { Forward: 5'-AGAGAAGATTAGCATGGCCC } \\ & \text { CTG-3' } \\ & \text { Reverse:5'-AGTGCAGGGTCCGAGGTATT-3' }\end{array}$

miRNA/miR, microRNA; RT-qPCR, reverse transcription-quantitative polymerase chain reaction.

identify the miRNA expression patterns of the patients with $\mathrm{CM}$. We made a heat map to visualize the results of the two-way hierarchical clustering of the miRNA (Fig. 1). The color scale shown at the top illustrated the relative expression level of a miRNA: Red represented a high relative expression level, whereas green represented a low relative expression level (20).

Of the 207 miRNAs analyzed by miRNA microarray, only four were identified as being differentially expressed by more than two-fold. Of those, 2 (miR-320a and miR-1249-5p) were obviously up-regulated and 2 (miR-634 and miR-6870-3p) were down-regulated in the patients with $\mathrm{CM}$ in comparison with the healthy controls $(\mathrm{P}<0.05$; Table II).

Validation of the differentially expressed miRNAs by $R T-q P C R$. To validate the microarray results, miR-320a and miR-634 were selected for further validation in the serum of the $30 \mathrm{CM}$ patients and 30 healthy controls by using RT-qPCR due to no target genes of miR-1249-5p and miR-6870-3p were predicted. Results showed that the expression of miR-320a increased and the expression of miR-634 decreased in the patients with CM in comparison with the normal controls $(\mathrm{P}<0.05)$. These results were consistent with the results of the microarray (Fig. 2).

Prediction of miR-320a target genes. The miRNA target genes were predicted using three databases including miRanda, TargetScan and PicTar. We identified 487 target genes from these three databases and only genes rank in the top 21 that were predicted for miR-320a are shown in Table III, and the remaining 466 genes are not shown in the table. However, following GO enrichment analysis and pathway enrichment analysis are conducted on the basis of these 487 genes.

GO enrichment analysis for the target genes of miR-320a. As shown in Fig. 3, the GO functional enrichment results revealed that the miR-320a target genes were enriched in numerous bioprocesses such as cell adhesion, cellular process, multicellular organismal development, and cellular component organization or biogenesis. The target genes were also involved in the synthesis of many cellular components, such as cell junction and extracellular matrix component. Moreover, the target genes was closely related to many molecular functions, such as necleic acid binding transcription factor activity.
Pathway enrichment analysis of the miR-320a target genes. On the basis of the classification of the GO annotation, the KEGG database was used to analyze the signaling pathways modulated by the target genes. As shown in the Fig. 4, the miR-320a target genes were mainly enriched in the bone morphogenetic protein (MBP) signaling pathway, nicotinamide adenine dinucleotide (NAD) pathway, de novo ceramide biosynthetic pathway and 3-phosphoinositide biosynthesis pathway (Fig. 4).

\section{Discussion}

miRNAs are a series of short (typically 18-25 nucleotides), single-stranded and highly conserved non-coding RNA molecules. They negatively regulate gene expression either through inhibition of mRNA translation or by promoting mRNA degradation. Emerging evidence suggests that miRNAs can be emerge as important intervention targets and predictive tools for various human cancers because of their stability and convenience of miRNA detection $(21,22)$. In particular, serum miRNAs may be used as biomarkers in diagnosis (6). Zhang et al (23) found that the expression of miR-217 is downregulated in tissues of CM patients, and overexpression of miR-217 inhibits the proliferation and promotes the apoptosis of the primary CM cells. Cao et al (24) reported that downregulation of miR-218 promotes the proliferation of cells derived from patients with CM. These findings suggested that miR-217 and miR-218 are potential targets for CM prevention and therapy due to their tumor suppression properties. However, the study on miRNA expression in the serum of patients with CM has not yet been reported. The present study reported for the first time the differential expression profiles of miRNA in the serum of patients with CM. We confirmed four differentially expressed miRNAs. Of these four miRNAs, the expression levels of miR-320a and miR-1249-5p increased and those of miR-634 and miR-6870-3p decreased in patients with CM in comparison with the healthy controls. Only four differentially expressed miRNAs were identified was due to large dispersion caused by the small sample size of our study. In a study conducted by Liu et al (25), sixteen patients were selected and their serum miRNA profiles were determined using a microarray, and the results showed that only four serum miRNAs were found to be differentially altered, it is consistent with our experimental results. Due to no target genes of miR-1249-5p and miR-6870-3p were predicted, only miR-320a and miR-634 were selected for further verification using RT-qPCR, and the results were consistent with the microarray results, such finding indicated the high reliability of the chip technology.

A newly found tumor suppressor, miR-634 plays an important role in inducing the apoptosis of tumor cells. miR-634 inhibits the cell proliferation and induces apoptosis in cervical cancer cells (26) and nasopharyngeal carcinoma cells (27). Moreover, Fujiwara et al (28) found that miR-634 enhanced chemotherapy-induced cytotoxicity in a model of esophageal squamous cell carcinoma. In the present study, the expression of miR-634 was lower in the serum of CM patients, suggesting that it may also be an important tumor suppressor in CM. This finding provided the basis for the further study of the role of miR-634 in CM.

In recent years, the differential expression of miR-320a between tumor tissue and healthy tissue was identified using 


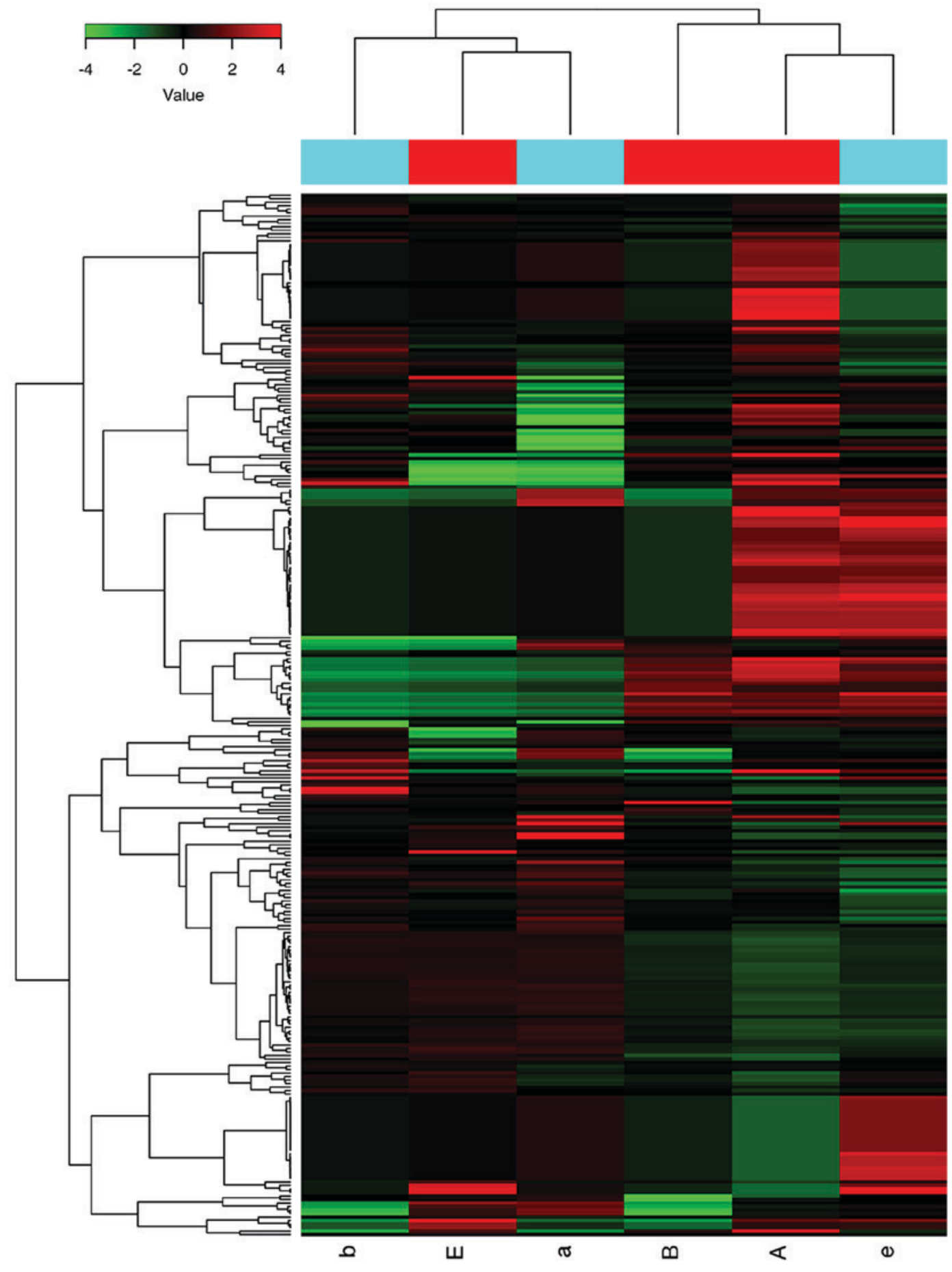

Figure 1. Heat map of microarray expression data from serum samples of patients with CM and normal controls. Upregulated miRNAs were indicated by red while downregulated miRNAs were indicated by green. A, B and E, CM patient serum samples; a, b and e, healthy control patient serum samples. CM, cardiac myxoma.

qPCR, gene chips and Western blot. Previous studies have revealed that miR-320a exhibits abnormal expression levels in multiple malignancies and is involved in the formation, progression and metastasis of cancer. miR-320a is down-regulated in various cancers such as breast cancer (29), bladder cancer (30), gastric cancer (31), colorectal cancer (32), and nasopharyngeal cancer (33). The overexpression of miR-320a suppresses the capability of cell migration and invasion and induces G0/G1 growth arrest (32). However, miR-320a does not exert an inhibition effect on all cancers. Yao et al (34) found that the overexpression of miR-320a promoted the migration and invasion of the hepatocellular carcinoma (HCC) cell line SMMC-7721, but decreased the expression of the endogenous $\mathrm{miR}-320 \mathrm{a} / \mathrm{c} / \mathrm{d}$ with specific inhibitors significantly inhibiting the migration and invasion of SK-Hep-1 cells. Wen et al (35) reported that miR-320a is markedly overexpressed in the HCC patients and could be serve as preclinical biomarkers. $\mathrm{Xu}$ et al (36) reported that miR-320a is upregulated in prostate cancer cells, and may exhibit an oncogenic function in prostate cancer. Recently, Xu et al (37) found that miR-320a 
Table II. Differentially expressed miRNA in the serum of cardia myxoma patients.

\begin{tabular}{lcrrc}
\hline Systematic name & P(Corr) & P-value & FC (abs) & Regulation \\
\hline hsa-miR-320a & 0.96 & 0.032 & 2.05 & $\mathrm{Up}$ \\
hsa-miR-1249-5p & 0.96 & 0.028 & 11.08 & $\mathrm{Up}$ \\
hsa-miR-634 & 0.96 & 0.047 & 5.47 & Down \\
hsa-miR-6870-3p & 0.96 & 0.049 & 5.97 & Down \\
\hline
\end{tabular}

FC (abs) is the absolute value of the expression difference between the two groups. P-values were calculated through the unpaired t-test method. P (Corr) is the correcting P-value that calculated through false discovery rate method. miR, microRNA.

Table III. Some of genes that were predicted for miR-320a.

\begin{tabular}{ll}
\hline miRNA & \multicolumn{1}{c}{ Target genes } \\
\hline miR-320a & CDH2, CPD, IGF1R, MAT2A, PBX3, TAF5, \\
& NRP1, IGF2BP3, USP25, ZFP91, TMEM47, \\
& MTDH, DNER, HECTD2, MIER3, ARF1, EREG, \\
& ESRRG, HOXA5, MAP1B, MN1
\end{tabular}

miRNA/miR, microRNA.

is a potentially valuable biomarker for diagnosing older females with gastric cancer. The difference expression levels in different cancers are associated with tumor types, tumor size, clinical stage and lymphatic metastasis (38).

Several studies on miR-320a in various cancers have been conducted. However, no studies have investigated the clinicopathological value of miR-320a expression in CM. In the present study, the expression of miR-320a in CM patients was higher than that in the healthy controls. This finding indicated that miR-320a may be an oncogene in CM.

miR-320a is important in a number of cancer types (39-41), and miR-320a was upregulated in this study, hence, we predicted its target genes, and identified 487 target genes using miRanda, TargetScan and PicTar. Of these target genes, myocyte enchancer factor 2D (MEF2D) is invovled in regulating tumor biology of $\mathrm{CM}$, it related to proliferation, invasion and tumor size of myxoma (42). VEGFA, which possesses a highly conserved binding site to miR-320a (43), probably induces angiogenesis for tumor growth in CMs $(44,45)$. Therefore, we assume that miR-320a is probably involved in tumor growth in CM by targeting VEGFA and MEF2D. Future research are needed to investigate this possibility. Moreover, the GO functional enrichment results showed that the major functional terms of the 487 target genes included cell adhesion, cellular process, multicellular organismal development, cellular component organization or biogenesis (biological process category), cell junction, extracellular matrix component (cellular component category) and nucleic acid binding transcription factor activity (molecular function category), This result indicated that some target genes regulated CM development in a joint manner.

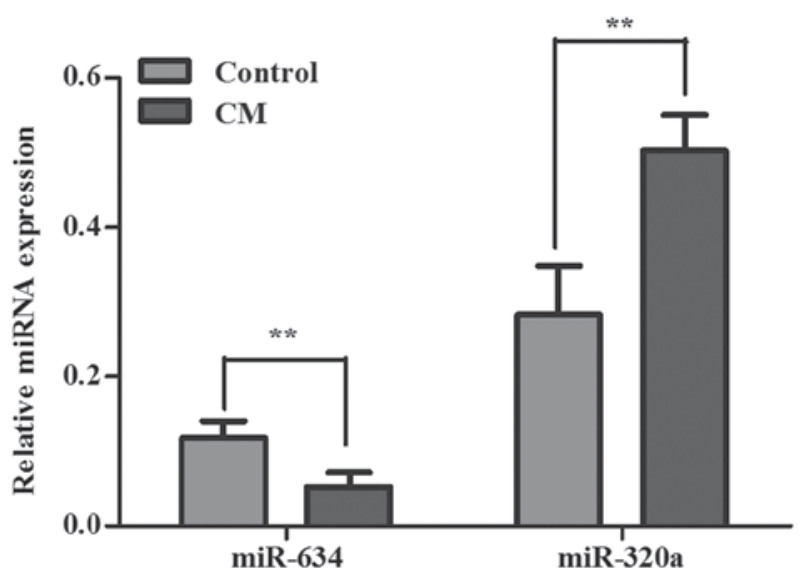

Figure 2. Validation of the expression of miR-320a and miR-634 in serum of CM patients $(n=30)$ and healthy controls $(n=30)$ detected by RT-qPCR. Data are presented as mean \pm standard deviation. ${ }^{* *} \mathrm{P}<0.01$. Control, healthy controls; CM, CM patients; RT-qPCR, reverse transcription-quantitative polymerase chain reaction.

The results of the pathway enrichment analysis showed that the target genes of miR-320a are mainly involved in the MBP signaling pathway, nicotinamide adenine dinucleotide (NAD) pathway, de novo ceramide biosynthetic pathway and 3-phosphoinositide biosynthesis pathway. Among these, de novo ceramide biosynthetic pathway is one of the most extensively studied pathways in cancer. As a powerful tumor suppressor, ceramide is a kind of sphingomyelin molecule that produced from sphingolipid metabolism or de novo synthesis when cells encounter extracellular signals and receptors such as TNF- $\alpha$, radiation and anticancer agents (46-51). It can act as the second messenger of various signal transduction pathways involved in the regulation of cell proliferation, differentiation and apoptosis through the activation of c-Jun nh2-terminal kinase (JNK), mitogen-activated protein kinase (MAPK), kinase suppressors of Ras (KSR) and other signaling pathways, and also can through effector molecules such as protein kinases, protein phosphatase 1 and protein phosphatase 2A (51-54). However, deficiencies in the ceramide production in cancer cells lead to the survival of tumour cells and resistance to chemotherapy (55). The apoptotic signaling pathways mediated by ceramide have been considered as targets for anticancer therapies (51). Therefore, miR-320a may be involved in $\mathrm{CM}$ development by through the ceramide signaling pathway. More of the differentially expressed miRNAs we identified participated in the ceramide pathway. Further studies are needed to elucidate the key pathways for the development and maintenance of CM.

We should note that there are some limitations in this study. First, this study has a relatively small sample size and future studies with larger cohorts should be conducted in the future. Second, due to limited funding for experiment, serum pools were produced for microarray analysis. This method will be unable to detect variations between individuals due to miRNA expression varies greatly between individuals. Moreover, it is impossible to perform a correlation analysis between the qPCR and microarray results because of pooling the samples. Third, only miR-320a and miR-634 were validated due to no target genes of 


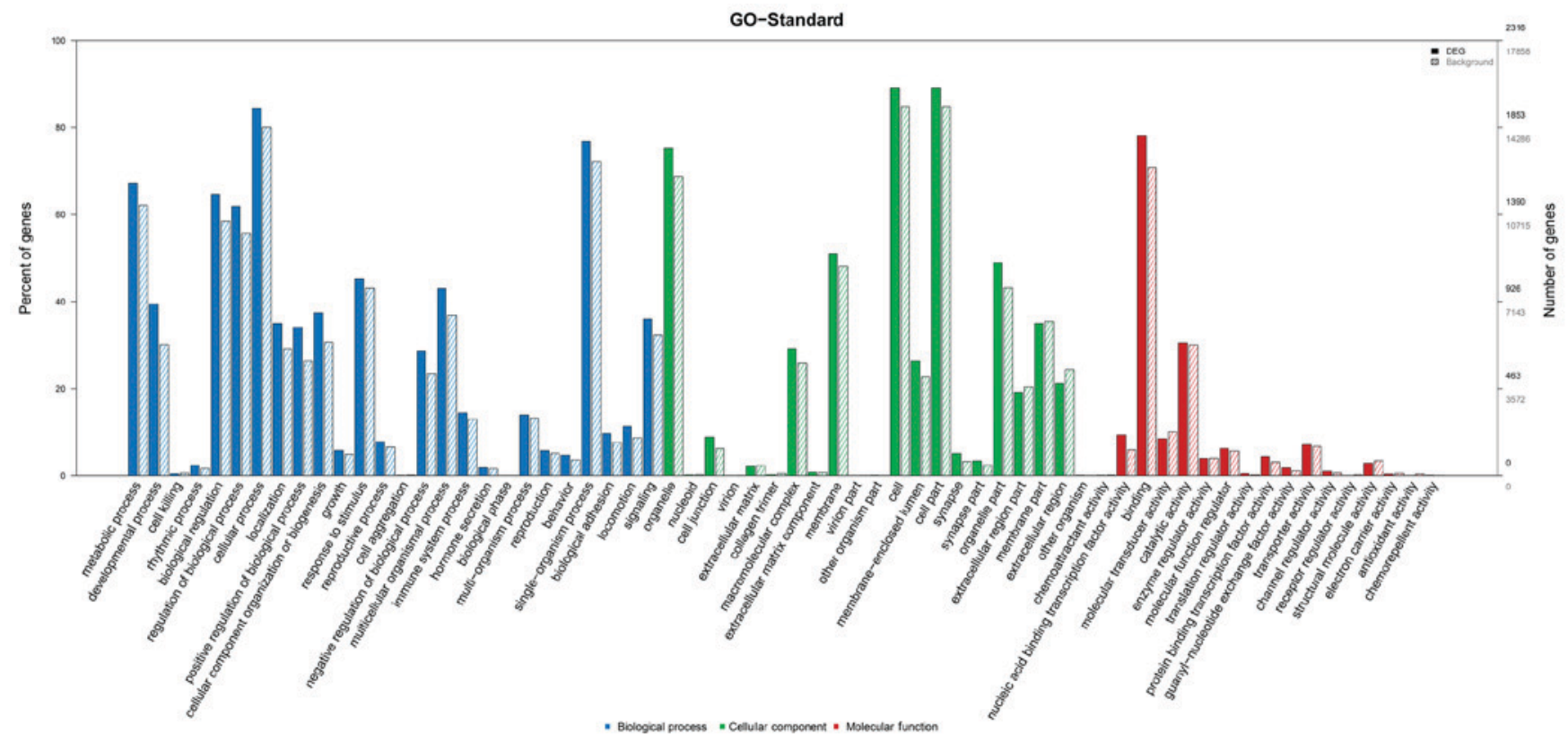

Figure 3. Major GO terms of miR-320a target genes in three GO categories (biological process, cellular component, and molecular function). The ordinate represents the ratio of 'the number of some genes involved in a GO term' to 'the total number of genes involved in all terms of one GO category (removal redundancy)' for every major GO term in the three GO categories. Data from the Gene Ontology (GO) database (http://www.geneontology.org/).

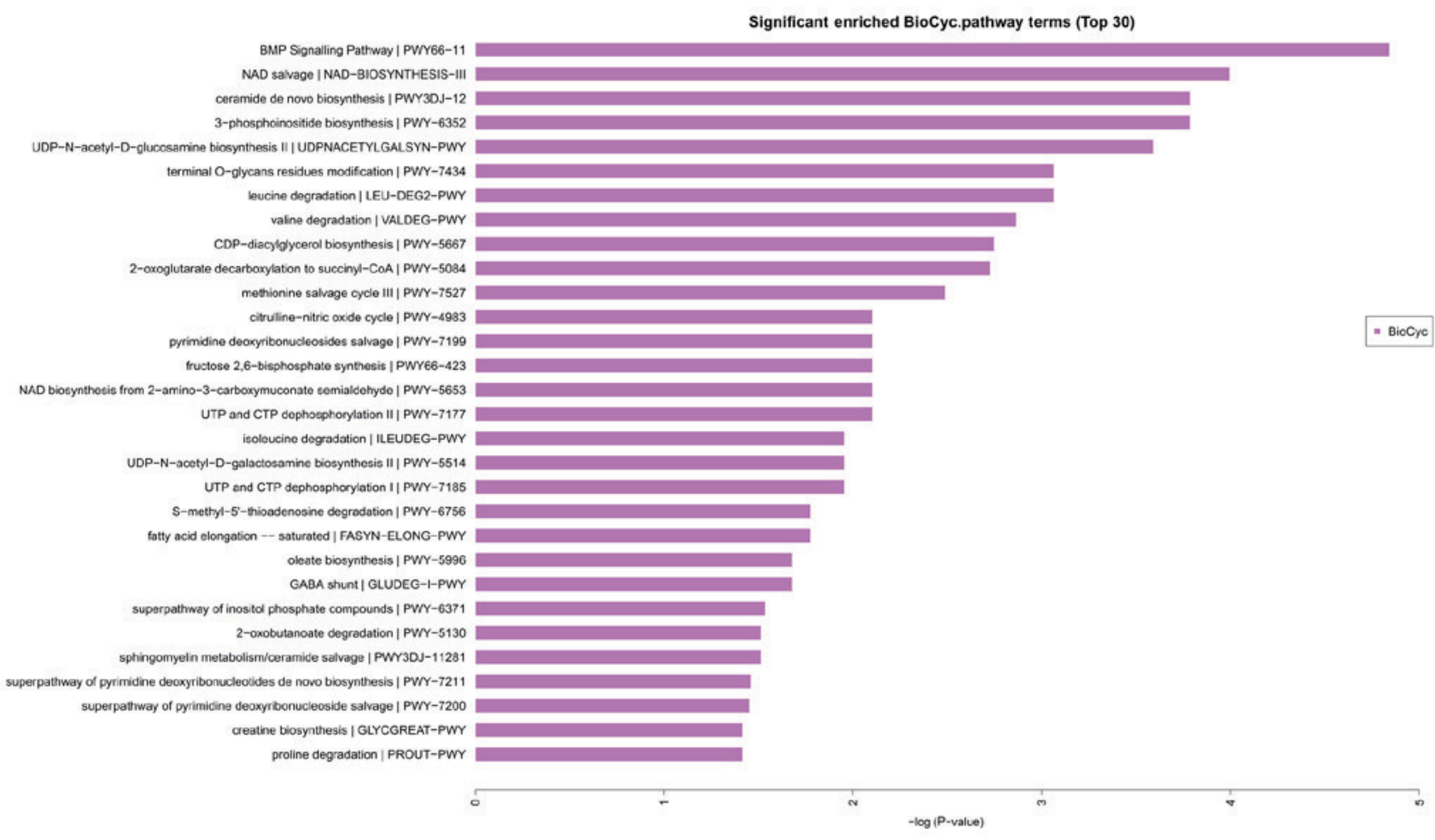

Figure 4. KEGG enrichment result of miR-320a predicted target genes. Data from the KEGG database (http://www.kegg.jp/).

miR-1249-5p and miR-6870-3p were predicted. Target genes of miRNA prediction relys on bioinfomatic algorithms. We used 11 softwares (miRWalk, Microt4, miRanda, mirbridge, miRDB, Pictar, PITA, miRMap, RNA22, RNAhybrid, Targetscan) to predict the targer genes of miR-1249-5p and miR-6870-3p, and found less than 4 softwares successfully predicted the targer genes of them. In our study, we chose the three most commonly used predicting softwares
(miRanda, TargetScan and PicTar) to study the target genes of differently expressed miRNA, but no targer genes of miR-1249-5p and miR-6870-3p were predicted by these three softwares, this will not have impacts on the reliability of our results. Fourth, there are few references on miRNA in tissue of CM patients (23), and our study reported for the first time the differential expression profiles of miRNA in the serum of patients with CM, so it's not easy to write 
incisive disccusion, unless the research goes further, and it is also not enough to affirm the validity of the two differently expressed miRNA detected in our study as markers for $\mathrm{CM}$ pathology, the association only remain proved. Fifth, we did not found that CM pathology associated to other miRNA serum markers related to miRNA we have found. The meaning of our study is to provide an experimental basis for the further exploration of exploring the specific miRNA for the diagnosis and treatment of CM. Finally, Common practice is to find differently expressed miRNAs and then verified them between patients and healthy people. The miRNA differential expression profile in CM patients remains unexplored, so we only study differently expressed miRNAs between CM patients and healthy people in our study. As the research moves along, we will tempted a selective analysis between man and women.

In conclusion, we reported for the first time the circulating miRNA profile of patients with CM and found that the expression levels miR-320a and miR-1249-5p were up-regulated and the expression levels of miR-634 and miR-6870-3p were down-regulated in CM patients. miR-320a is mainly involved in the cellular physiological reaction and possibly participates in $\mathrm{CM}$ development through the ceramide signaling pathway. The results in this study provided an experimental basis for the further exploration of exploring the specific miRNA for the diagnosis and treatment of CM.

\section{Acknowledgements}

The authors would like to thank Zhihao Yang for his assistance with the data analysis in this study.

\section{Funding}

This study was supported by grants from the key project of young and middle-aged backbone talents cultivation of of Fujian Province Health System of China (No. 2015-ZQN-ZD-16).

\section{Availability of data and materials}

The datasets used and analyzed during the current study are available from the corresponding author on reasonable request.

\section{Authors' contributions}

JL carried out sample preparation, and experimental validation. QW and LY performed the experiments, analyzed the data, and wrote the manuscript. LC conceived of the study and participated in its design and coordination. All authors read and approved the final manuscript.

\section{Ethics approval and consent to participate}

Ethical approval was given by the Ethics Committee of The Union Hospital of Fujian Medical University (No. 2017KY027).

\section{Consent for publication}

Not applicable.

\section{Competing interests}

The authors declare that they have no competing interests.

\section{References}

1. Debourdeau P, Gligorov J, Teixeira L, Aletti M and Zammit C: Malignant cardiac tumors. Bull Cancer 91 (Suppl 3): S136-S146, 2004 (In French).

2. Lee KS, Kim GS, Jung Y, Jeong IS, Na KJ, Oh BS, Ahn BH and Oh SG: Surgical resection of cardiac myxoma-a 30-year single institutional experience. J Cardiothorac Surg 12: 18 , 2017.

3. Mazzurin AV, Soboleva NI and Semenov BN: Cardiac myxomas in children. Pediatriia 46: 50-53, 1967 (In Russian).

4. Butany J, Nair V, Naseemuddin A, Nair GM, Catton C and Yau T: Cardiac tumors: Diagnosis and management. Lancet Oncol 6: 219-228, 2005.

5. Reynen K: Cardiac myxomas. New Engl J Med 333: 1610-1617, 1995.

6. Jain D, Maleszewski JJ and Halushka MK: Benign cardiac tumors and tumorlike conditions. Ann of Diagn Pathol 14: 215-230, 2010.

7. Rupp GM, Heyman RA, Martinez AJ, Sekhar LN and Jungreis CA: The pathology of metastatic cardiac myxoma. Am J Clin Pathol 91: 221-227, 1989.

8. Bartel DP: MicroRNAs: Target recognition and regulatory functions. Cell 136: 215-233, 2009.

9. Garzon R, Fabbri M, Cimmino A, Calin GA and Croce CM: MicroRNA expression and function in cancer. Trends Mol Med 12: 580-587, 2006.

10. Jansson MD and Lund AH: MicroRNA and cancer. Mol Onco 6: 590-610, 2012.

11. Chan SH, Wu CW, Li AF, Chi CW and Lin WC: miR-21 microRNA expression in human gastric carcinomas and its clinical association. Anticancer Res 28: 907-911, 2008.

12. Iorio MV, Ferracin M, Liu CG, Veronese A, Spizzo R, Sabbioni S, Magri E, Pedriali M, Fabbri M, Campiglio M, et al: MicroRNA gene expression deregulation in human breast cancer. Cancer Res 65: 7065-7070, 2005.

13. Chen X, Ba Y, Ma L, Cai X, Yin Y, Wang K, Guo J, Zhang Y, Chen J, Guo X, et al: Characterization of microRNAs in serum: A novel class of biomarkers for diagnosis of cancer and other diseases. Cell Res 18: 997-1006, 2008.

14. Zhu Z, Qi Y, Ge A, Zhu Y, Xu K, Ji H, Shi Z, Cui L and Zhou M: Comprehensive characterization of serum microRNA profile in response to the emerging avian influenza A (H7N9) virus infection in humans. Viruses 6: 1525-1539, 2014.

15. Krawczynski K, Najmula J, Bauersachs S and Kaczmarek MM: MicroRNAome of porcine conceptuses and trophoblasts: Expression profile of micrornas and their potential to regulate genes crucial for establishment of pregnancy. Biol Reprod 92: 21, 2015.

16. Shen J, Xiao Z, Wu WK, Wang MH, To KF, Chen Y, Yang W, $\mathrm{Li}$ MS, Shin VY, Tong JH, et al: Epigenetic silencing of miR-490-3p reactivates the chromatin remodeler SMARCD1 to promote Helicobacter pylori-induced gastric carcinogenesis. Cancer Res 75: 754-765, 2015.

17. Ho CY, Bar E, Giannini C, Marchionni L, Karajannis MA, Zagzag D, Gutmann DH, Eberhart CG and Rodriguez FJ: MicroRNA profiling in pediatric pilocytic astrocytoma reveals biologically relevant targets, including PBX3, NFIB, and METAP2. Neuro Oncol 15: 69-82, 2013.

18. Li X, Peng B, Zhu X, Wang P, Xiong Y, Liu H, Sun K, Wang $\mathrm{H}$, Ou L, Wu Z, et al: Changes in related circular RNAs following ER $\beta$ knockdown and the relationship to rBMSC osteogenesis. Biochem Biophys Res Commun 493: 100-107, 2017.

19. Livak KJ and Schmittgen TD: Analysis of relative gene expression data using real-time quantitative PCR and the 2(-Delta Delta C(T)) method. Methods 25: 402-408, 2001.

20. Eisen MB, Spellman PT, Brown PO and Botstein D: Cluster analysis and display of genome-wide expression patterns. Proc Natl Acad Sci USA 95: 14863-14868, 1998.

21. Kong YW, Ferland-McCollough D, Jackson TJ and Bushell M: microRNAs in cancer management. Lancet Oncol 13: e249-e258, 2012. 
22. Corsini LR, Bronte G, Terrasi M, Amodeo V, Fanale D, Fiorentino E, Cicero G, Bazan V and Russo A: The role of microRNAs in cancer: Diagnostic and prognostic biomarkers and targets of therapies. Expert Opin Ther Targets 16 (Suppl 2): S103-S109, 2012.

23. Zhang J, Wang $\mathrm{C}$ and $\mathrm{Xu} \mathrm{H}$ : miR-217 suppresses proliferation and promotes apoptosis in cardiac my xoma by targeting Interleukin-6. Biochem Biophys Res Commun 490: 713-718, 2017.

24. Cao Q, Dong P, Wang Y, Zhang J, Shi X and Wang Y: miR-218 suppresses cardiac myxoma proliferation by targeting myocyte enhancer factor 2D. Oncol Rep 33: 2606-2612, 2015.

25. Liu N, Cui RX, Sun Y, Guo R, Mao YP, Tang LL, Jiang W, Liu X, Cheng YK, He QM, et al: A four-miRNA signature identified from genome-wide serum miRNA profiling predicts survival in patients with nasopharyngeal carcinoma. Int J Cancer 134: 1359-1368, 2014.

26. Cong J, Liu R, Wang X, Jiang H and Zhang Y: MiR-634 decreases cell proliferation and induces apoptosis by targeting mTOR signaling pathway in cervical cancer cells. Artif Cells Nanomed Biotechnol 44: 1694-1701, 2016.

27. Peng X, Cao P, He D, Han S, Zhou J, Tan G, Li W, Yu F, Yu J, Li Z and Cao K: MiR-634 sensitizes nasopharyngeal carcinoma cells to paclitaxel and inhibits cell growth both in vitro and in vivo. Int J Clin Exp Pathol 7: 6784-6791, 2014.

28. Fujiwara N, Inoue J, Kawano T, Tanimoto K, Kozaki K and Inazawa $\mathrm{J}$ : miR-634 activates the mitochondrial apoptosis pathway and enhances chemotherapy-induced cytotoxicity. Cancer Res 75: 3890-3901, 2015.

29. Wang B, Yang Z, Wang H, Cao Z, Zhao Y, Gong C, Ma L, Wang X, Hu X and Chen S: MicroRNA-320a inhibits proliferation and invasion of breast cancer cells by targeting RAB11A. Am J Cancer Res 5: 2719-2729, 2015.

30. Shang C, Zhang H, Guo Y, Hong Y, Liu Y and Xue Y: MiR-320a down-regulation mediates bladder carcinoma invasion by targeting ITGB3. Mol Biol Rep 41: 2521-2527, 2014.

31. Zhu Y, Zhang Y, Sui Z, Zhang Y, Liu M and Tang H: USP14 de-ubiquitinates vimentin and miR-320a modulates USP14 and vimentin to contribute to malignancy in gastric cancer cells. Oncotarget 8: 48725-48736, 2017

32. Zhao H, Dong T, Zhou H, Wang L, Huang A, Feng B, Quan Y, Jin R, Zhang W, Sun J, et al: miR-320a suppresses colorectal cancer progression by targeting Rac1. Carcinogenesis 35: $886-895,2014$

33. Qi X, Li J, Zhou C, Lv C and Tian M: MicroRNA-320a inhibits cell proliferation, migration and invasion by targeting BMI-1 in nasopharyngeal carcinoma. FEBS Lett 588: 3732-3738, 2014

34. Yao J, Liang LH, Zhang Y, Ding J, Tian Q, Li JJ and He XH: GNAI1 suppresses tumor cell migration and invasion and is post-transcriptionally regulated by $\mathrm{Mir}-320 \mathrm{a} / \mathrm{c} / \mathrm{d}$ in hepatocellular carcinoma. Cancer Biol Med 9: 234-241, 2012.

35. Wen Y, Han J, Chen J, Dong J, Xia Y, Liu J, Jiang Y, Dai J, Lu J, Jin G, et al: Plasma miRNAs as early biomarkers for detecting hepatocellular carcinoma. Int J Cancer 137: 1679-1690, 2015.

36. Xu G, Wu J, Zhou L, Chen B, Sun Z, Zhao F and Tao Z: Characterization of the small RNA transcriptomes of androgen dependent and independent prostate cancer cell line by deep sequencing. PLoS One 5: e15519, 2010.

37. Xu Q, Dong QG, Sun LP, He CY and Yuan Y: Expression of serum miR-20a-5p, let-7a, and miR-320a and their correlations with pepsinogen in atrophic gastritis and gastric cancer: A case-control study. BMC Clin Pathol 13: 11, 2013.
38. Yang HP, Yu J, Wang L, Ding D, Zhang L, Chu C, Chen Q, $\mathrm{Xu} \mathrm{Z}$, Zou Q and Liu X: miR-320a is an independent prognostic biomarker for invasive breast cancer. Oncol Lett 8: 1043-1050, 2014.

39. Diakos C, Zhong S, Xiao Y, Zhou M, Vasconcelos GM, Krapf G, Yeh RF, Zheng S, Kang M, Wiencke JK, et al: TEL-AML1 regulation of survivin and apoptosis via miRNA-494 and miRNA-320a. Blood 116: 4885-4893, 2010.

40. Hummel R, Wang T, Watson DI, Michael MZ, Van der Hoek M, Haier J and Hussey DJ: Chemotherapy-induced modification of microRNA expression in esophageal cancer. Oncol Rep 26: 1011-1017, 2011.

41. Salendo J, Spitzner M, Kramer F, Zhang X, Jo P, Wolff HA, Kitz J, Kaulfuß S, Beißbarth T, Dobbelstein M, et al: Identification of a microRNA expression signature for chemoradiosensitivity of colorectal cancer cells, involving miRNAs-320a, -224, -132 and let7g. Radiother Oncol 108: 451-457, 2013.

42. Huo Y, Zhao Q, Wang C, Zhao F, Du Y and Sun W: The involvement of myocyte enhancer factor $2 \mathrm{D}$ in regulating tumor biology of cardiac myxoma. Tumour Biol 37: 5405-5411, 2016.

43. Yin Z, Zhao Y, Li H, Yan M, Zhou L, Chen C and Wang DW: miR-320a mediates doxorubicin-induced cardiotoxicity by targeting VEGF signal pathway. Aging (Albany NY) 8: 192-207, 2016.

44. Kono T, Koide N, Hama Y, Kitahara H, Nakano H, Suzuki J, Isobe $\mathrm{M}$ and Amano J: Expression of vascular endothelial growth factor and angiogenesis in cardiac myxoma: A study of fifteen patients. J Thorac Cardiovasc Surg 119: 101-107, 2000.

45. Amano J, Kono T, Wada Y, Zhang T, Koide N, Fujimori M and Ito K: Cardiac myxoma: Its origin and tumor characteristics. Ann Thorac Cardiovasc Surg 9: 215-221, 2003.

46. Hannun YA and Obeid LM: Ceramide: An intracellular signal for apoptosis. Trends Biochem Sci 20: 73-77, 1995.

47. Gulbins E: Regulation of death receptor signaling and apoptosis by ceramide. Pharmacol Res 47: 393-399, 2003.

48. Kolesnick R and Fuks Z: Radiation and ceramide-induced apoptosis. Oncogene 22: 5897-5906, 2003.

49. Ogretmen B and Hannun YA: Biologically active sphingolipids in cancer pathogenesis and treatment. Nat Rev Cancer 4: 604-616, 2004.

50. Modrak DE, Gold DV and Goldenberg DM: Sphingolipid targets in cancer therapy. Mol Cancer Ther 5: 200-208, 2006.

51. Lin CF, Chen CL and Lin YS: Ceramide in apoptotic signaling and anticancer therapy. Curr Med Chem 13: 1609-1616, 2006

52. Mathias S, Peña LA and Kolesnick RN: Signal transduction of stress via ceramide. Biochem J 335: 465-480, 1998.

53. Hannun YA and Obeid LM: Ceramide and the eukaryotic stress response. Biochem Soc Trans 25: 1171-1175, 1997.

54. Stoica BA, Movsesyan VA, Lea PM IV and Faden AI: Ceramide-induced neuronal apoptosis is associated with dephosphorylation of Akt, BAD, FKHR, GSK-3beta, and induction of the mitochondrial-dependent intrinsic caspase pathway. Mol Cell Neurosci 22: 365-382, 2003.

55. Morad SAF and Cabot MC: Ceramide-orchestrated signalling in cancer cells. Nat Rev Cancer 13: 51-65, 2013.

This work is licensed under a Creative Commons Attribution-NonCommercial-NoDerivatives 4.0 International (CC BY-NC-ND 4.0) License. 